\title{
A SOLAR AND GALACTIC COSMIC RAY SATELLITE EXPERIMENT**
}

\author{
William E. Althouse, Edward C. Stone, and Rochus E. Vogt \\ California Institute of Technology \\ Pasadena, California
}

and

Timothy H. Harrington

Analog Technology Corporation Pasadena, California

\section{Summary}

A cosmic ray instrument for NASA's OGO-F spacecraft is described. The experiment consists of three charged particle detector systems which are designed to measure the spectra and chemical composition of galactic and solar cosmic rays over selected energy intervals. Two of the detector systems, the $\triangle E$-Range and $\triangle E$-Cerenkov telescopes, will measure the galactic flux and smaller solar flare fluxes, while a third detector system, the Flare telescope, will measure larger fluxes.

The three telescopes identify particle energy and type by various techniques. Energy loss and range, which provide the identification of protons ( 1 to $300 \mathrm{MeV}$ ), alpha particles ( 4 to $1200 \mathrm{MeV}$ ) and electrons ( 1 to $500 \mathrm{MeV}$ ), are measured in a stack of silicon solid state detectors and tungsten absorbers in the $\triangle E$-Range telescope, while the identification of protons (350 to $1000 \mathrm{MeV}$ ), alpha particles (1400 to $4000 \mathrm{MeV}$ ) and nuclei through oxygen is provided in the $\Delta E$-Cerenkov telescope by energy loss measurements in silicon solid state detectors and a velocity measurement in a quartz Cerenkov radiator. The Flare telescope employs a double energy loss measurement in silicon detectors for the identification of protons (17 to $100 \mathrm{MeV}$ ) and alpha particles (70 to $400 \mathrm{MeV}$ ).

The design of the instrument is optimized for maximum use of the available data rate with minimum weight, volume and power requirements. This has been accomplished by the use of low-power integrated-circuit logic elements packaged in a dense yet flexible 3 -dimensional array, by the use of redundant data readout techniques to enhance data reliability, by the use of combined shift register-counter elements for both PHA address registers and event rate monitors, and by the use of timed overflow accumulators which count events for low rates and measure time-to-overflow for high rates.

* Work supported by the National Aeronautics and Space Administration under contract NAS5-9312 and grant NsG-426.
The instrument uses 72 bits of OGO's 1152bit main commutator data sequence; the transmitted data include event identification, pulse height analyzer data, range data, counting rates of all principle coincidences, all single detector rates, ground command status, and telescope temperatures. The instrument consumes 2.8 watts.

\section{Introduction}

A cosmic ray experiment with three charged particle telescopes will be flown by the California Institute of Technology aboard OGO-F, an Orbiting Geophysical Observatory of the National Aeronautics and Space Administration. The experiment will measure the energy spectra of charged particles of both solar and galactic origin. Due to the large dynamic range of the parameters measured, the required instrument is highly complex. It uses a number of different nuclear particle detectors in an arrangement optimized for the scientific objectives and the stringent requirements of observations in space.

\section{$\underline{\text { Scientific Objectives }}$}

The scientific objectives of the Caltech OGO-F experiment include the measurement of the energy spectra of electrons and nuclei with charge $\mathrm{Z}=1$ through $\mathrm{Z}=8$ in the cosmic radiation. The instrument is designed to allow a direct energy determination of electrons from about $1 \mathrm{MeV}$ to $500 \mathrm{MeV}$, protons and helium nuclei from $1 \mathrm{MeV}$ to $1 \mathrm{GeV} /$ nucleon, and 1 ithium through oxygen nuclei from $350 \mathrm{MeV} /$ nucleon to $1 \mathrm{GeV} /$ nucleon. The measurable differential energy range will be further extended to about $15 \mathrm{GeV} /$ nucleon by using the geomagnetic field as a spectral analyzer. Above $15 \mathrm{GeV} /$ nucleon the integral fluxes of the individual nuclei will be separated and recorded.

The sensitivity of the Caltech experiment will allow measurements of cosmic ray intensities from about $10^{-2}$ particles $/ \mathrm{m}^{2} \mathrm{sec}$ sr MeV to about $10^{10}$ particles $/ \mathrm{m}^{2} \mathrm{sec} \mathrm{sr}$, which covers the scientifically interesting range from quiet day galactic fluxes to the largest known solar flare particle injection. 
OGO-F will be placed in a polar orbit of about $82^{\circ}$ prograde inclination, with a perigee of $400 \mathrm{~km}$ and an apogee of $1300 \mathrm{~km}$. The spacecraft will be oriented with the Caltech telescopes at all times facing radially away from the earth. The orbital parameters of $0 \mathrm{GO}-\mathrm{F}$ are advantageous for the above measurements, since they allow observations of the total cosmic ray energy spectrum down to the lowest energies over the polar regions, where the geomagnetic cutoff is negligible, and allow use of the geomagnetic field as a spectral analyzer in its passage across lower geomagnetic latitudes.

The results of these observations will be used in studies of the astrophysical aspects of cosmic radiation and the radiation environment of the earth, including:

1. Solar modulation of galactic cosmic ray intensities.

2. Acceleration and injection of high energy particles by the sun, and their storage in the interplanetary fields.

3. Solar induced changes in the earth's magnetosphere, deduced from modifications in geomagnetic cutoffs.

4. Solar proton fluxes which produce polar cap absorption.

The scheduled 1968 launch date falls into a period of high solar activity, when modulation effects and solar high energy particle production will be most pronounced.

\section{The Detector System}

In order to meet the scientific objectives a detector system must have adequate charge and energy resolution, with a minimum of spurious (background) events, and must be operative over a large range of incident intensities. In addition, the polar orbit poses definite restrictions on the type of rate analysis employed and upon the degree of experiment control which is possible by ground command.

The restrictions on the rate measurements arise from the rapid motion of the spacecraft in the geomagnetic field, resulting in moderate changes in counting rate due to changing geomagnetic cutoffs and large changes due to trapped particles. Key rates are averaged over $430 \mathrm{msec}$ intervals, during which time the spacecraft has moved only $\sim 1.5$ min in latitude.

The charge $Z$ and the energy $E$ of cosmic ray particles may be determined by an appropriate combination of measurements. For example, it is possible, within certain restrictions, to measure total energy, energy loss, range, velocity, or rigidity. Due to the broad coverage of incident energies and fluxes desired, no single, feasible combination of measurements appeared optimum. For this reason, the instrument includes three different, complementary counter telescopes (labeled $\triangle E$-Range, $\triangle E$-Čerenkov, and Flare), each optimized over more restricted energy and flux intervals, with built-in priorities so that ground commands are not necessary for routine operation.

\section{The Electronic System}

The electronic system for this experiment consists of eight major functional parts: 1) the Range telescope electronics; 2) the Č telescope electronics; 3) the Flare telescope electronics; 4) the analog processing electronics; 5) the detector coincidence, anti-coincidence, and priority logic; 6) the rate accumulator subsystem; 7) the data storage, formatting, readout, and spacecraft interface electronics; and 8) the system power converter. These functions are arranged as shown in the system block diagram, Fig. 1.

The telescope assemblies are electrically and mechanically separate, each containing its own solid state detectors, active and/or passive collimators, and amplifier and discriminator circuitry. Careful layout, grounding, and integral mounting techniques insure that negligible crosstalk exists between signal paths.

The remaining electronic circuitry is contained in a separate box. The coincidence and priority logic and the rate accumulators serve each telescope as required, while the analog processor and data storage section are time-shared between the telescopes. The $\Delta E$-Range and $\Delta E$ Cerenkov telescopes are handled on an equal priority basis, while the Flare telescope is given absolute priority at all times. A maximum of one event can be processed per readout cycle.

An event may originate in any of the three telescopes. The coincidence and priority logic checks the discriminator outputs to see if the event meets the response requirements for that telescope. If these conditions are met, then the event indicators and pulse height analyzer accumulators are reset, the new identification is written into storage, and the linear gates in the analog processor are opened to allow the delayed linear pulses into the three 255 channel pulse height analyzers. A toggle is set which blocks further events until after readout.

A rate accumulator subsystem continuously monitors twenty singles and coincidence rates. These rate measurements establish flux values within the telescopes and provide performance data on individual detectors so that faults may be rapidly identified and remedied.

A detector disabling system, controlled by ground command, minimizes the effects of failed detectors. This feature, coupled with the rate monitoring system, allows the experiment to continue operation with minimal data loss.

The data storage and readout section formats the experiment event and rate information into the spacecraft's synchronous data frame. All event identification and pulse height data are read out in a single, short data block, thus minimizing system dead time. If no new event occurs between 
readouts, the readout section repeats the event: data from the previous frame.

These major subsystems are described in detail below.

\section{The $\Delta E$-Range Telescope}

In the energy range from 1 to $300 \mathrm{MeV} /$ nucle:n for protons and alpha particles, a simultaneous measurement of the energy loss $\Delta E$ in a solid state detector and of the range in tungsten is employe: to identify both the charge and the energy of the incident particle. A cross section of the $\Delta E-$ Range telescope is shown in Fig. 2. The energy loss $\triangle E$ is measured in each of the silicon solid state detectors D1, D2 and D3, while the range of the incident particle is determined by the penetration of detectors D4 through D7 which are sandwiched between the tungsten absorbers A3 through A6. The entire detector-absorber stack is completely surrounded, except for the entrance and exit apertures, by a plastic scintillator anti-coincidence cup D8 which has the dual function of collimating acceptable events and rejecting background events due to nuclear interactions and side showers. The collimation results in a geometrical factor $(A \Omega)$ which is a function of range, varying from $1.5 \mathrm{~cm}^{2}$ sr down to $0.19 \mathrm{~cm}^{2}$ sr, as shown in Fig. 2. The top of the collimator is covered with a 0.00015 " thick aluminized mylar light baffle.

The response of the telescope to protons and alpha particles is shown in Fig. 3, in which the average energy loss $(\triangle E)$ in $D 1$ and $D 2$ and the range are plotted as a function of incident energy. Note that particles with a given range can be assigned a unique energy interval and can be identified by their $\Delta E$ as a proton or alpha particle. Electrons can also be identified by $\Delta E$, but a corresponding energy interval cannot be as accurately assigned due to the statistical nature of the electron cascade process.

Figure 4 shows a functional block diagram of the $\triangle E$-Range telescope electronics. Each solid state detector is connected to a charge sensitive preamplifier using bipolar transistors in a con-. ventional cascode design. Each preamplifier is followed by a shaping amplifier with single integration-single differentiation $0.5 \mu \mathrm{s}$ RC pulse: shaping. Conversion gain for D1 is 0.5 volts/ $\mathrm{MeV}$; conversion gain for D2 through D7 is 0.67 volts/MeV.

Mu1tiple $\triangle E$ measurements in $D 1, D 2$, and $D 3$ identify valid events and permit the reduction of effects due to statistical fluctuations, nuclear interactions, and spurious background events. D1, D2, and D3 each have a single threshold discriminator-trigger which generates a $3.5 \mu \mathrm{s}$ pulse. Range and additional $\Delta E$ measurements in the form of two discriminators on each detector D4 through D7 are employed to uniquely separate a stopping low energy proton from electrons and from more energetic protons, which might undergo nuclear interactions.
The D8 signal chain consists of a charge sensitive amplifier with single differentiation $\mathrm{RC}$ shaping ( $1 \mu \mathrm{s}$ time constant) and a fast recovery threshold discriminator-trigger which generates a $4.0 \mu \mathrm{s}$ pulse.

The choice of detectors was dictated by the requirements of thermal-vacuum stability, maximum immunity to radiation damage, and suitable depletion depths and areas. Detectors D2 through D7 are nominally $1000 \mu$ thick, $4.5 \mathrm{~cm}^{2}$, totally depleted surface barrier devices. The wafer size of $\mathrm{D} 1$ is also $4.5 \mathrm{~cm}^{2}$, but the thickness is only $100 \mu$, totally depleted, and the sensitive area is $2.5 \mathrm{~cm}^{2}$ as defined by the gold evaporation. The sma11 thickness of D1 insures relative insensitivity to electrons and allows protons with as little energy as $3 \mathrm{MeV}$ to penetrate to D2.

\section{The $\triangle E$-Čerenkov Telescope}

In the energy range from 350 to $1000 \mathrm{MeV} /$ nucleon, a simultaneous measurement of $\Delta E$ in a solid state detector and Cerenkov (C) radiation in the $1 \mathrm{~cm}$ thick quartz window of a photomultiplier tube is employed to identify the charge (from $\Delta E$ ) and the velocity (from $C$ ) of the incident particle. A cross section of the $\Delta E$-Cerenkov telescope is shown in Fig. 5. The energy loss $\triangle E$ is measured in both $\mathrm{D}^{\prime}{ }^{\prime}$ and $\mathrm{D} 2{ }^{\prime}$, which are nominally $1000 \mathrm{p}$ thick, $4.5 \mathrm{~cm}^{2}$, totally depleted surface barrier detectors. Collimation is again provided by a plastic scintillator anticoincidence shield $\mathrm{D} 4^{\prime}$, resulting in a geometrical factor of $2.8 \mathrm{~cm}^{2} \mathrm{sr}$. The aperture is covered with a $0.00075^{\prime \prime}$ thick aluminized mylar sheet.

The response of the $\Delta E$-Čerenkov telescope is shown in Fig. 6, in which the most probable $\triangle E$ in $D 1^{\prime}$ (or $D 2^{\prime}$ ) is plotted as a function of relative light output from C-radiation in the quartz window. In order to take advantage of the directional character of Č-radiation, the top (upward facing) surface and sides of the quartz window are painted black, so that only particles entering the telescope from above will fully illuminate the photocathode.

A functiona1 block diagram of the $\triangle E$-Čerenkov telescope is shown in Fig. 7. $1^{\prime}{ }^{\prime}$ and $\mathrm{D} 2{ }^{\prime}$ are connected to charge sensitive amplifiers with pulse height analyzed dynamic ranges of $~ 350$ (150 keV to $54 \mathrm{MeV}$, see Fig. 6). This is accomplished by producing a non-1inear'transfer characteristic for these detectors.

The preamplifier output drives an amplifier with precision gain and saturation characteristics, both of which are determined by stable feedback components. The output of this amplifier and the output of the preamplifier are then summed passively and fed into a shaping amplifier identical to that used in the $\Delta E$-Range telescope. The resultant output pulse has constant shaping ( $0.5 \mu \mathrm{s}$ rise- and fall-time constants), and a two section piecewise-linear transfer function yielding $20 \mathrm{keV}$ per channel up to $2.5 \mathrm{MeV}$ (channel 125), and $400 \mathrm{keV}$ per channel from $2.5 \mathrm{MeV}$ 
to $54.5 \mathrm{MeV}$ (channe1 255).

The Cerenkov signal is treated in a similar manner, with typically $0.033 \mathrm{pC}$ per channe1 below $6 \mathrm{pC}$ (channel 180), and $0.915 \mathrm{pC}$ per channel between $6 \mathrm{pC}$ and $88 \mathrm{pC}$ (channel 255). One picocoulomb typically corresponds to a relative light output of 1.0 in Fig. 6.

\section{The Flare Telescope}

During large solar flare events the plastic scintillatorsused as collimators for the $\Delta E-$ Range and $\triangle E$-Cerenkov telescopes will be counting at a rate which exceeds the electronic capability of the system $\left(>10^{5} \mathrm{sec}^{-1}\right)$, resulting in improper event analysis. For this reason, a smal1, twodetector, passively collimated counter telescope is included in the experiment. A cross section of the telescope is shown in Fig. 8, schematical1y indicating the copper collimator-shield surrounding $\mathrm{D}^{\prime}{ }^{\prime}$ and $\mathrm{D} 6^{\prime}$, which are nominally $1000 \mu$ thick, $0.2 \mathrm{~cm}^{2}$ area, totally depleted surface barrier detectors. The energy loss $\triangle E$ is measured in both detectors, with the response of $\mathrm{D} 5^{\prime}$ and D6' being essentially identica1 to D2 (see Fig. 3) and D3 respectively. The copper collimator-shield is designed to reduce the single detector counting rates by stopping all protons with less than $100 \mathrm{MeV}$ energy.

Analysis of Flare telescope events is assured by a priority system which preferentially accepts and holds these events for readout. The geometrical factor $\left(0.02 \mathrm{~cm}^{2} \mathrm{sr}\right)$ and therefore the analysis rate, has been chosen such that when the incident flux is large enough to saturate the $\triangle E$-Range and $\triangle E$-Cerenkov systems, the readout of Flare analyses occupies about one-half of the experiment readout capability. Larger fluxes will result in the readout of proportionately more Flare telescope events.

A block diagram of the Flare telescope electronics is shown in Fig. 9. Treatment is identical to that of the $\triangle E$-Range telescope, except that fast-recovery discriminator-triggers are used.

\section{$\underline{\text { Analog Processor }}$}

A block diagram of the analog processing subsystem is shown in Fig. 10. The analog processor contains three independent pulse-height-to-time converters, associated gated clocks, and PHA address storage/readout registers. The pulse-height converter circuitry is shared among the three telescopes under control of the coincidence and priority logic subsystem. Eight detectors from the three telescopes are pulse-height analyzed; Table I 1 ists these detectors and the energy loss corresponding to channel 255 .

The $2 \mu$ s delay-line in each signal path provides time for the logic system to accept or reject a given event and to open the appropriate linear gates. The linear gates, which are of the shunt type, are open for the threshold discriminator period, $3.5 \mathrm{\mu s}$. The coincidence and prior- ity logic allows only one linear gate to each pulse-height converter to be opened at one time. The linear gate outputs are fed into a "mixer" circuit which simply reproduces at its output the signal at any of its inputs. Provision for scale factor adjustment is also made in the mixer circuit.

The output of the mixer circuit is connected into the pulse-height-to-time converter, which generates a logic pulse whose width is proportiona1 to the peak amplitude of the input pulse. This logic pulse then gates $\mathrm{ON}$ a $1 \mathrm{MHz}$ delay-line clock which produces a burst of self-completing pulses, the number of which is proportional to the energy loss in the detector which initiated the event.

The gated clock is converted into a binary word by the gated clock accumulators. In addition to the counting function, the accumulators, which can be reset in $1 \mu \mathrm{s}$ by the priority logic, contain overflow logic and function as output shift registers. The counter used to accomplish these functions is an 8-bit, length 255 shiftcode pattern generator, which is a shift register with logical feedback to the input stage from other stages in the register. Because the counter is a shift register, readout is accomplished by disabling the logical feedback and gating readout shift pulses into the counter, thus shifting out the data in the register.

The particular generator chosen for this application is shown in Fig. 11. State 254 is detected by the overflow gate, and the next pulse, the 255 th, sets the overflow flip-flop. This f1ip-flop causes al1-zero readout, uniquely identifying the occurrence of an overflow event.

\section{Coincidence and Priority Logic}

Operation of the entire instrument is controlled by the coincidence and priority logic. This subsystem, acting upon the discriminator pulses from the three telescopes, determines which events are to be processed and which are to be rejected. A block diagram of this section is shown in Fig. 12.

The outputs of the telescope discriminatortrigger circuits are transformer-coupled into the coincidence and priority logic. The time coincidence of certain of these outputs determines which events may be accepted. From the $\triangle E$-Range telescope, either D1 D8 or D2D3D8 represents a good event. The function $\mathrm{D} 1^{\prime} \mathrm{D} 2{ }^{\prime} \mathrm{D} 3^{\prime} \mathrm{D} 4{ }^{\prime}$ is required

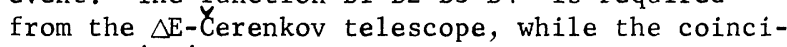
dence $\mathrm{D} 5$ ' $\mathrm{D} 6$ ' is required to process events from the Flare telescope. If an event meets one of these coincidence requirements, the linear gates corresponding to that telescope's analog signals are opened in the analog processor.

The occurrence of an acceptable event triggers a system reset pulse which resets the event identification storage and pulse height accumulators. New event identification is written into storage; if the event occurred in the $\triangle E$-Range 
telescope, pulses from the range detectors D4 through D7 are stored.

An event from the $\triangle E$-Range or the $\triangle E-$ Cerenkov telescopes sets a toggle which blocks further events from these telescopes, but allows an event from the Flare telescope. When an event occurs in the Flare telescope, a second toggle, called priority inhibit, blocks all three telescopes. These two toggles are reset after readout.

A network of six latching relays, controlled by ground command, provides for modification of the coincidence logic in the event of detector failure (e.g., due to radiation damage). Either $\mathrm{D} 1$, D2, or D3 may be independent1y disabled in the $\Delta E$-Range telescope, while either $\mathrm{D} 1^{\prime}$ or $\mathrm{D} 2{ }^{\prime}$ may be disabled in the $\triangle E$-Cerenkov telescope. Any of the three telescopes may be disabled entirely by removing power from that telescope.

\section{Rate Accumulator Subsystem}

The rates of the pulses produced by the discriminators and coincidence logic are monitored by seven rate accumulators. Six of the accumulators monitor the coincidence signa $1 \mathrm{~s}$ D1 $\overline{\mathrm{D}} 8$, D2 $\overline{\mathrm{D}} 8$, $\mathrm{D} 2 \mathrm{D} 3 \overline{\mathrm{D}} 8, \mathrm{D} 1{ }^{\prime} \mathrm{D} 2{ }^{\prime} \mathrm{D} 3{ }^{\prime} \mathrm{D} 4^{\prime}$, and $\mathrm{D} 5{ }^{\prime} \mathrm{D} 6{ }^{\prime}$, and the $\mathrm{D} 5{ }^{\prime}$ singles rate. The first four rates are each sampled over three spacecraft data frames, while D5' and D5 'D6' are sampled for one frame. The seventh accumulator, connected to a sixteen position commutator, monitors a11 14 individual detector rates except $\mathrm{D} 5{ }^{\prime}$, and the coincidence rate $\mathrm{D} 1 \mathrm{D} 2 \overline{\mathrm{D}} 8$. The rate accumulators accommodate event rates ranging from near zero to about $10^{4}$ per second. The low event rates are counted directly to allow accumulation of total events over an interval greater than a single sampling interval, while high event rates are compressed so that telemetry bits will be efficiently used. This is accomplished by using a dual-mode counting system.

The dual-mode counter accumulates events as a simple scaler until overflow occurs. After overflow, the scaler counts clock pulses. Since the sampling interval, the overflow clock rate, and the counter overflow point are known, the average event rate may be determined. The overflow clock rate is selected so that it cannot cause a second overflow. Since certain rates are sampled for one spacecraft data frame while the others are sampled for three, two different overflow clock rates are used. By deriving the overflow clocks from the spacecraft-supplied shift pulses, they are automatically adjusted for variations in spacecraft bit rate, thus optimizing counting resolution for various sampling intervals.

A logic diagram of the accumulator is shown in Figure 13. The scaler is a simplified version of the 8-bit shift-code pattern generator used for gated clock accumulation. The simplification is in the use of 2-tap feedback instead of the 4-tap version used before. This produces a length 217 pattern instead of length 255, but reduces the feedback gating from 8 gates to 2 .
A oneshot in the shift line prevents ambiguous operation if a data pulse is coincident with the edge of a write signal.

\section{Spacecraft Electrical Interface}

The instrument output data is both analog (0-5 VDC) and digital (serial, NRZ). The spacecraft telemetry system is a PCM system with 128 9-bit words per frame, transmitted at a nominal rate of seven frames per second. One of these words is further subcommutated by a 128-position subcommutator. This instrument is assigned the digital data words 9, 10, 11, 12, 113, and 114 and analog words 39 and 87 in the main frame, and subcommutated words 72 (digital), and 87 and 106 (analog). The word format is shown in Figure 14, which also shows the instrument subcommutation sequence for words 113 and 114, and the variable format for the event data. An experiment generated odd parity bit is included as the ninth bit of each digital word.

The digital data are transferred to the spacecraft on a single line in response to word gate signals, one for each output word, lasting for 9 bit times. The word pulse is synchronized to the shift pulses so that it is sufficient to gate the shift pulses into a 9 -bit shift register with the appropriate word signal and use that same word signal to gate the output of the register to the telemetry line.

Redundant data-handling systems are provided by the spacecraft, requiring a dual interface and a selection circuit to inhibit the unused data output, word gate, and shift pulse lines.

Analog output data is of two forms. Logarithmic ratemeters connected to discriminators D8 and $\mathrm{D} 4{ }^{\prime}$ are read out as analog words 39 and 87 respectively, with sensitivities of 1 volt per decade $\left(1 \mathrm{~V}=10 \mathrm{sec}^{-1}, 5 \mathrm{~V}=10^{5} \mathrm{sec}^{-1}\right)$. Temperature-sensitive resistors in the $\triangle E$-Range and $\Delta E$-Cerenkov telescopes are read out in subcommutated analog words 87 and 106 respectively.

\section{Event Data}

Digital words 9 through 12 contain data pertaining to individual events. Word 9 contains four bits identifying the event type, and provides the key for decoding words 10, 11, and 12. Also included in word 9 are overflow bits from the three accumulators which can be read out in a frame, and a new-data bit to indicate if the information in words 9 through 12 has changed since the previous readout.

Data are destructively written into this section of the readout system. A Range, Flare, or Cerenkov event indicator pulse from the coincidence and priority logic is used to clear the system, and to set one of the four bits in word 9 . These bits control which of three optional word 10 registers are to be read out. In the case of a Flare telescope event, the D5'D6' rate accumula- 
tor is read out. If a Range event occurs with any of the range detectors D4 through D7 triggered, 8 range bits corresponding to the two discriminators on each of the detectors D4 through D7 are read out. If neither of these occur, the gated-clock accumulator for HTC 1 will be read out. The source for the signal analyzed by HTC 1 , as wel1 as those for HTC 2 and HTC 3 read out in words 11 and 12 , is controlled by the coincidence and priority logic.

\section{Rate and Housekeeping Data}

The remaining digital words 113,114 and 72 present data in a fixed format. Words 113 and 114 are connected via a 3-position commutator to six rate accumulators, one of which, 114-3, is connected to a 16-position commutator on its input. Subcommutator word 72 reads out the state of the six command relays and the position of the 113-114 commutator once each 128 frames.

The accumulator read out in word 114-3 meaures 13 detector rates, one coincidence rate, and counts the fast overflow clock. The remaining position of the 16 position commutator provides synchronization. Monitoring the fast overflow clock verifies both overflow clock systems, since it will cause overflow in the 114-3 accumulator at a known time, after which the slow overflow clock will be counted. Synchronization is provided by forcing a data output of all zeros, a forbidden state of this accumulator.

\section{Power Supply}

The low-voltage power supply for this instrument is a regulator followed by a squarewave switching mode static inverter and transformerrectifier system. The input voltage ranges from 23.5 to 33.5 volts. The output voltages are regulated by comparing the $+12 \mathrm{~V}$ to a low temperature coefficient Zener diode and controlling the switching-mode regulator on the input side of the system with the amplified difference. The switching mode regulator and the static inverter are synchronized to a spacecraft-provided power synchronization signal, although free-run capability also exists. Protective devices include reverse-polarity protection and an active current limiter, set well below the trip level of the spacecraft power line fuses.

Circulating ground currents and power converter noise are controlled by providing good capacitive and resistive isolation.

The high-voltage for the phototubes is obtained with a tuned class-C oscillator and voltage multiplier enclosed within a feedback loop which compares the high voltage output with a stable reference.

\section{Instrument Construction}

Discrete components in a planar layout are used for the amplifiers and discriminators on the telescopes and in the high voltage sections of the power supply; soldered cordwood modules and low power integrated circuits are used for the remaining electronics. The instrument uses approximately 450 discrete transistors and 260 integrated circuit flatpacks. Nominal power consumption is 2.8 watts with all three telescopes operating. A photograph of the instrument is shown in Figure 15.

\section{Acknowledgements}

The amplifier and discriminator circuits, the gates used in the coincidence and priority logic, and the analog circuitry in the analog processor were designed by the Laboratory for Applied Sciences (now the Laboratory for Astrophysics and Space Research) of the University of Chicago.

The instrument was funded by the National Aeronautics and Space Administration; development and construction was carried out under contract NAS5-9312, with partial development funding by NASA Grant NsG-426. We wish to express our appreciation for this support.

Table I

Pulse Height Analyzed Detectors and Energy Loss Corresponding to Full Scale

\begin{tabular}{lccc} 
Telescope & HTC 1 & HTC 2 & HTC 3 \\
\cline { 2 - 3 } & $\mathrm{D} 1$ & $\mathrm{D} 2$ & $\mathrm{D} 3$ \\
& $14^{\mathrm{MeV}}$ & $12 \mathrm{MeV}$ & $12 \mathrm{MeV}$ \\
$\Delta$-Čerenkov $^{\prime}$ & $\mathrm{D}^{\prime}$ & $\mathrm{D} 2^{\prime}$ & $\mathrm{D} 3^{\prime}$ \\
Flare & $54^{\mathrm{MeV}}$ & $54^{\mathrm{MeV}}$ & $88^{\mathrm{pC}}$ \\
& - & $\mathrm{D} 5^{\prime}$ & $\mathrm{D} 6^{\prime}$ \\
& & $12 \mathrm{MeV}$ & $12 \mathrm{MeV}$
\end{tabular}




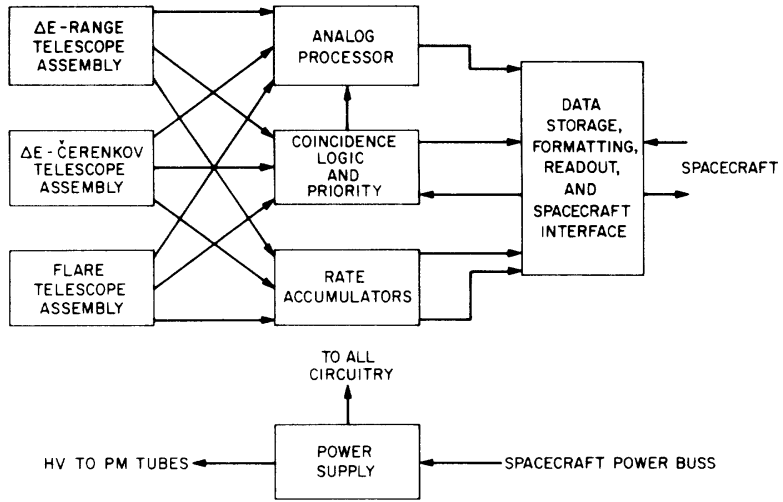

Fig. 1. Electronic system block diagram

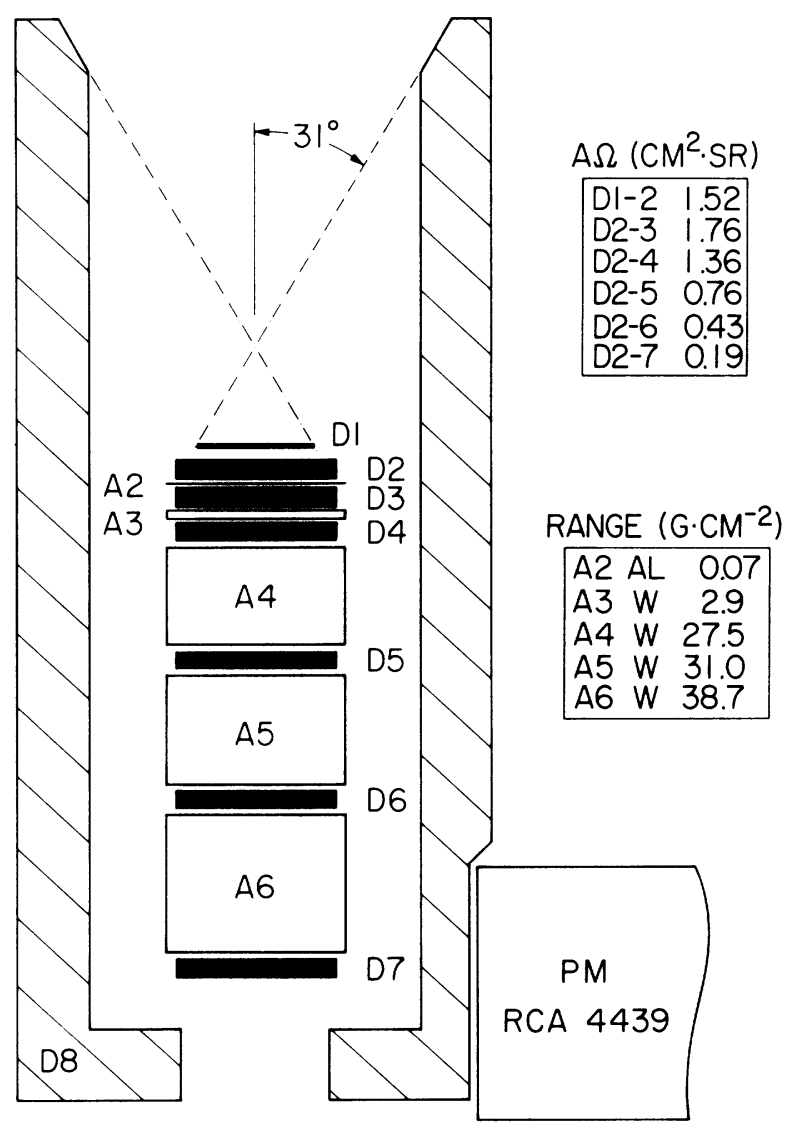

Fig. 2. Cross-section of E-Range telescope

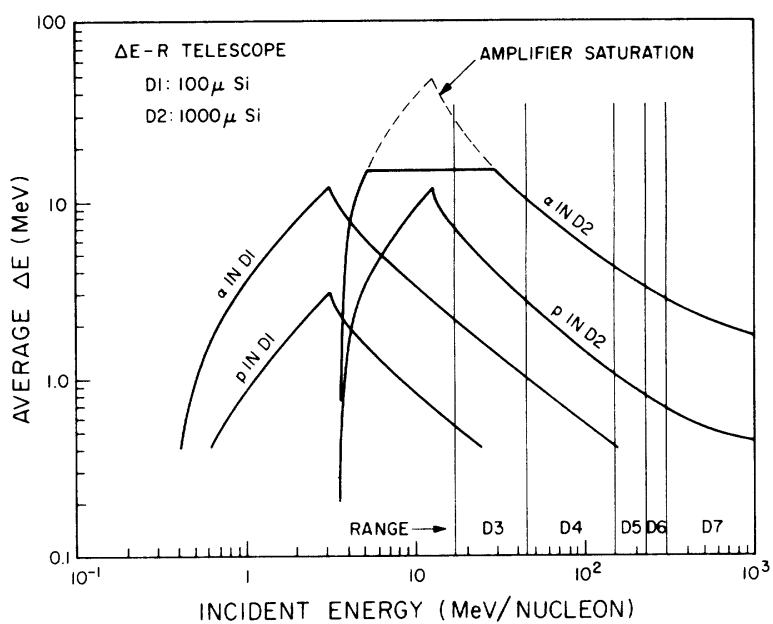

Fig. 3. Calculated response of E-Range telescope. Energy loss in detectors D1 and D2 is plotted vs. incident energy: vertical lines indicate range vs. incident energy as determined by penetration of detectors D3 through D7.

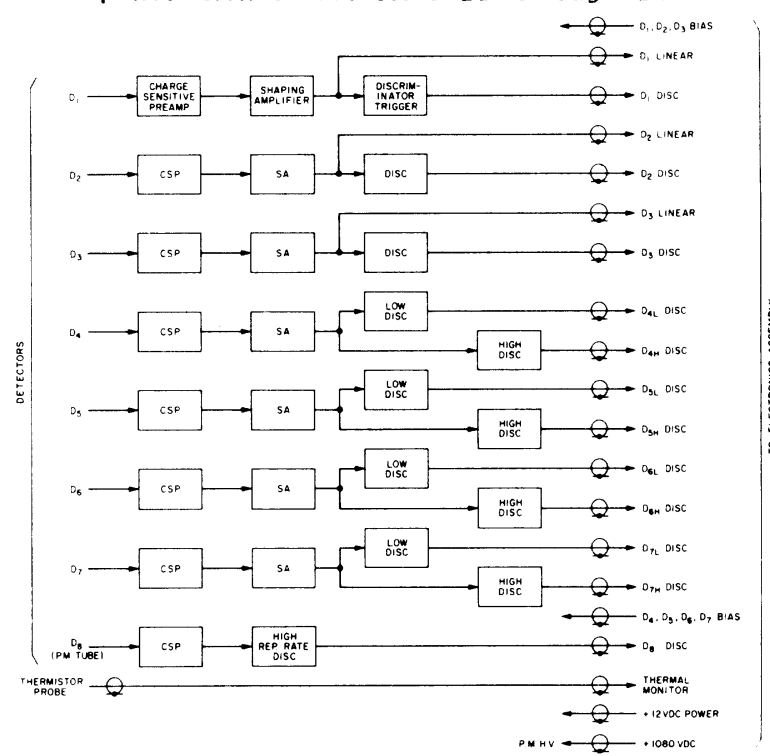

Fig. 4. Block diagram of E-Range telescope electronics

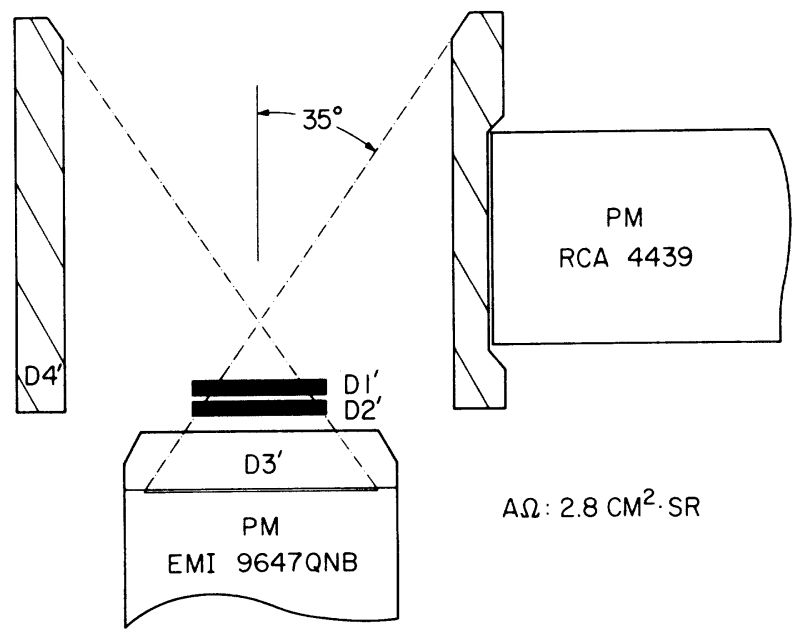

Fig. 5. Cross-section of E-Cerenkov telescope 


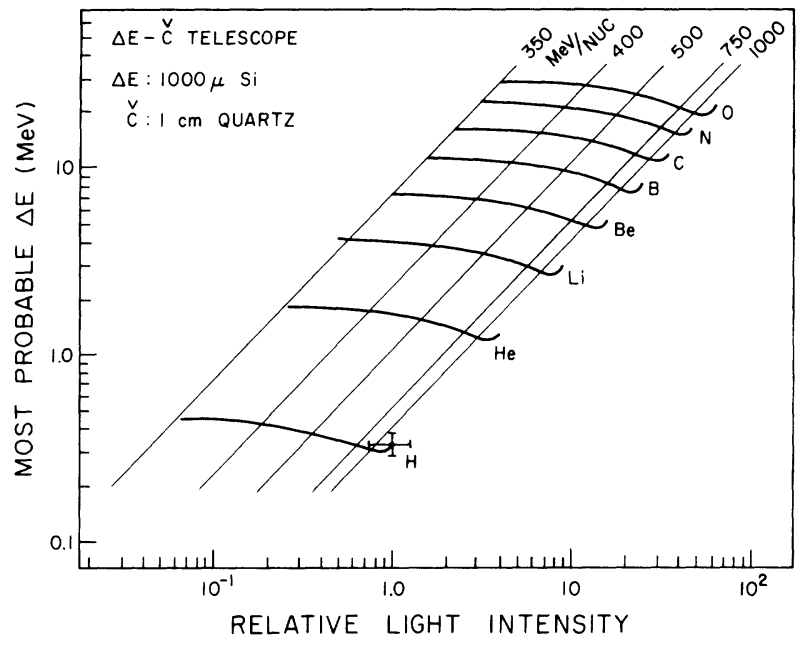

Fig. 6. Calculated response of E-Cerenkov telescope. Energy loss of nuclei ( $\begin{array}{ll}Z & 1\end{array}$ through $Z \quad 8$ ) in detectors D1' or D2 vs. relative light output from Cerenkov radiator. The diagonal lines indicate energy per nucleon.

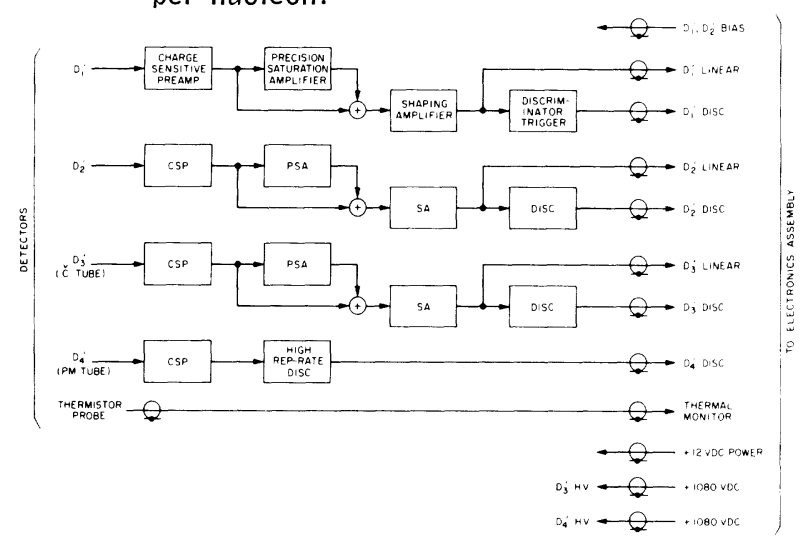

Fig. 7. Block diagram of E-Cerenkov telescope Electronics

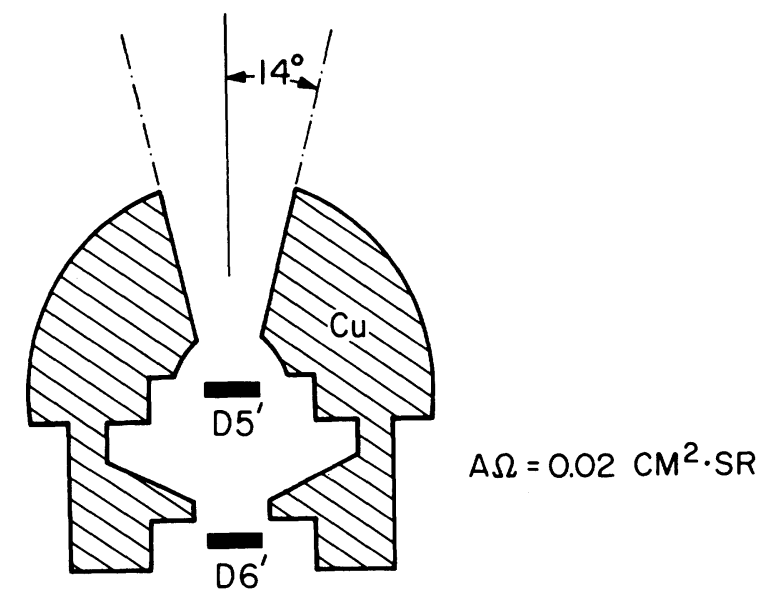

Fig. 8. Cross-section of the Flare telescope

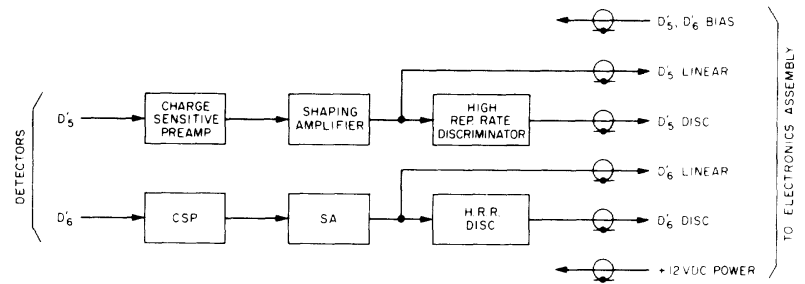

Fig. 9. Block diagram of Flare telescope electronics

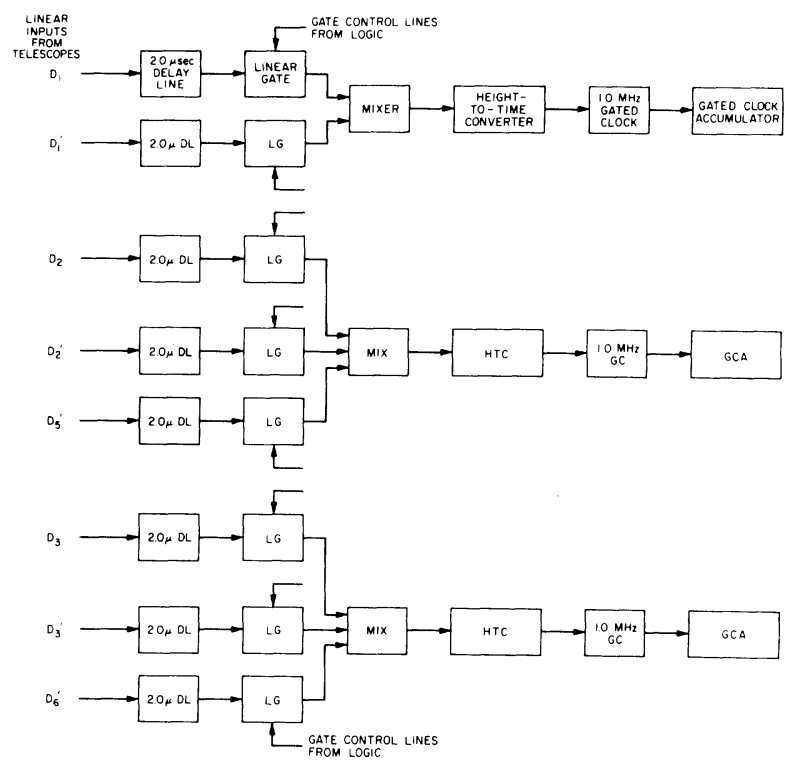

Fig. 10. Analog processing electronics block diagram

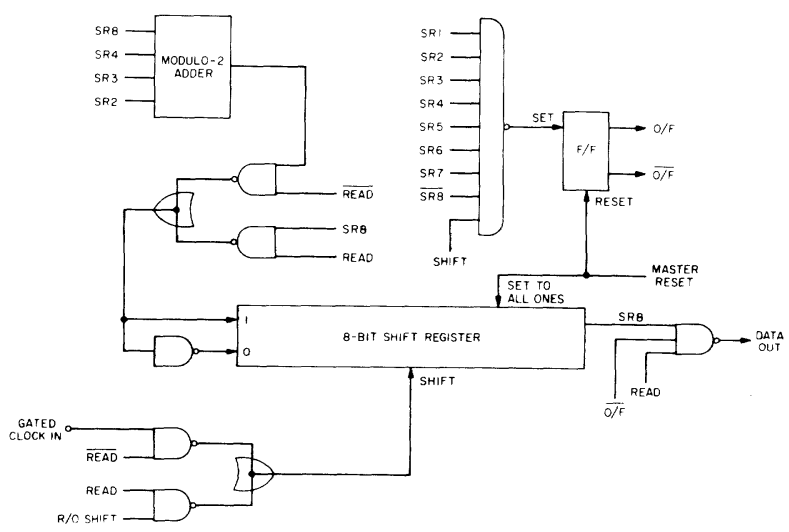

Fig. 11. Gated clock accumulator 


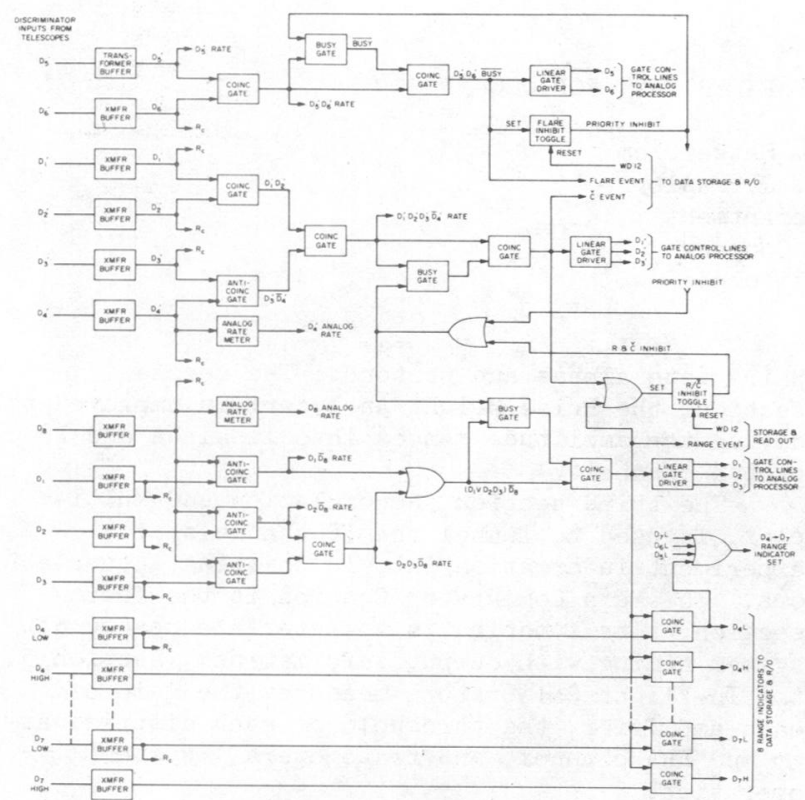

Fig. 12. Block diagram of the coincidence and priority logic

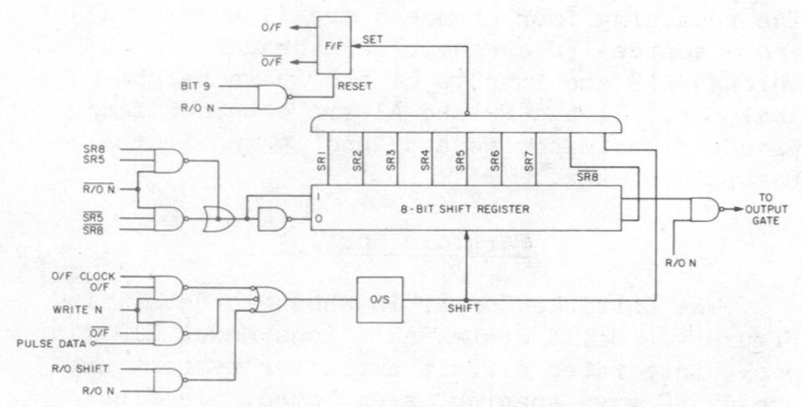

Fig. 13. Rate accumulator

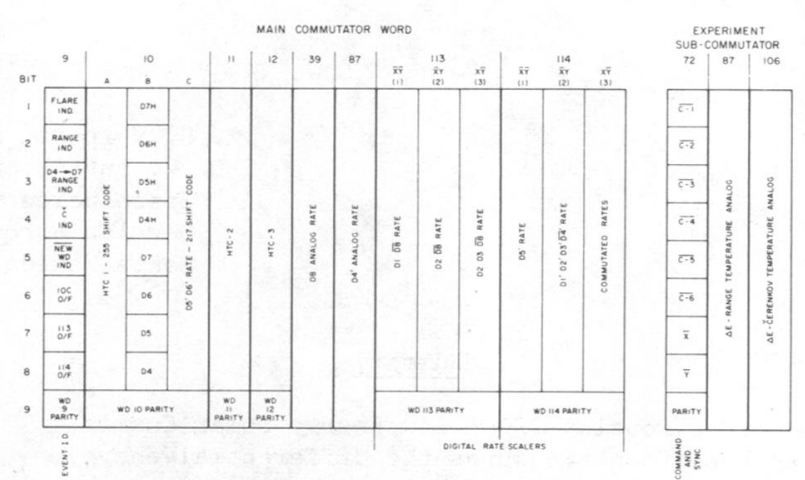

Fig. 14. Experiment data format

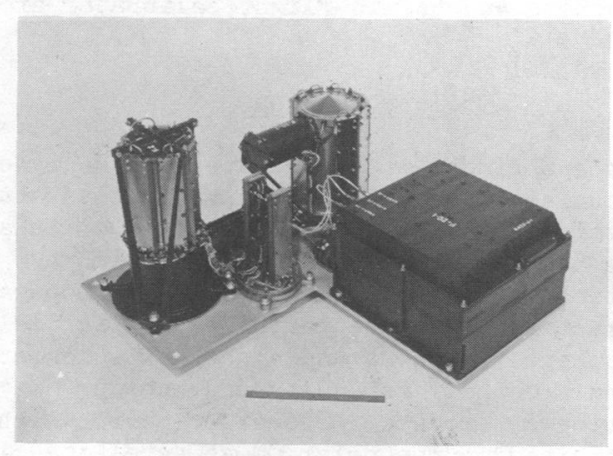

Fig. 15. Photo of the instrument. The E-Cerenkov telescope is on the left, the E-Range telescope in the rear. 